\title{
INFLUENCIA DE DIFERENTES INTENSIDADES DE DESRAMA SOBRE A PORCENTAGEM DE LENHO TARDIO E QUANTIDADE DE NÓS DA MADEIRA DE PRIMEIRO DESBASTE DE Pinus elliottii Engelman
}

\section{INFLUENCE OF DIFFERENT PRUNING INTENSITIES ON THE PERCENTAGE OF SUMMERWOOD AND QUANTITY OF KNOTS FROM FIRST THINNING OF Pinus elliottii Engelman}

Ana Cristina Schilling ${ }^{1}$ Paulo Renato Schneider ${ }^{2}$

Clóvis Roberto Haselein ${ }^{3}$ César Augusto Guimarães Finger ${ }^{4}$

\begin{abstract}
RESUMO
Nesse trabalho buscou-se avaliar a influência de diferentes intensidades de desrama sobre fatores de qualidade da madeira, como a porcentagem de lenho tardio e a quantidade de nós na madeira de primeiro desbaste de Pinus elliottii Engelman. Para tanto, foram utilizados dados de um experimento em blocos ao acaso com 3 repetições, instalado em povoamento com 10 anos de idade, onde foram avaliados 5 tratamentos: T1 - sem desrama; T2 - desrama seca; T3 - desrama de 40\% da altura total; T4 - desrama de 50\% da altura total; e T5 - desrama de 60\% da altura total. Em cada unidade experimental foram amostradas 3 árvores, das quais foram retiradas as duas primeiras toras e dois discos, a aproximadamente 0,10 e 2,5 $\mathrm{m}$ de altura. A partir das toras foram feitos corpos de prova com dimensão nominal de 0,04 × 0,08 × 2,5 m, utilizados na avaliação da quantidade de nós. A medição da porcentagem de lenho tardio foi feita sobre os discos. $\mathrm{Na}$ análise dos dados não foi encontrada regressão linear ou quadrática significativa, ao nível de $5 \%$ de significância, para nenhuma das variáveis estudadas. Da mesma forma, não se encontrou diferenças significativas entre as médias dos tratamentos.
\end{abstract}

Palavras-chave: desrama, qualidade da madeira, lenho tardio, nós.

\section{ABSTRACT}

This work was carried ou to evaluate the influence of different pruning intensities on some aspects of quality of wood (such as percentage of summerwood and quantity of knots) obtained

1. Eng. Floresal, M.Sc. em Manejo Florestal/CCR/UFSM. CEP:97105-900. Santa Maria. RS.

2. Eng. Florestal, Dr., Prof. Titular do Departamento de Ciências Florestais/CCR/UFSM. CEP: 97105-900. Santa Maria. RS.

3. Eng. Florestal, PhD., Prof. Adjunto do Departamento de Ciências Florestais/CCR/UFSM. CEP: 97105-900. Santa Maria. RS.

4. Eng. Florestal, Dr., Prof. Adjunto do Departamento de Ciências Florestais/CCR/UFSM. CEP: 97105-900. Santa Maria. RS. 
from first thinning of Pinus elliottii Engelman plantation forest. Random three-replication blocks were utilized, set on a ten-year old stand, where five treatments were analysed: T1 - with no pruning; T2 - dry pruning; T3 - pruning of 40\%; T4 - 50\%, and T5 - 60\% of the total canopy height, respectively. In each experimental unity, three trees were sampled. From each the first two logs were taken as well as two disks, at 0.10 and 2.5 metres of height. The logs were cut into $0.04 \mathrm{x}$ $0.08 \times 2.5$ metre nominal size beams and analysed the quantity of knots. The disks were used to determine the summerwood percentage. The data analysis with any of the variables studied did not show any significant linear regression, at a 5\% significance level. No significant differences were found among treatments means of the variables studied.

Key words: pruning, wood quality, summerwood, knots.

\section{INTRODUÇÃO}

Na década de 60, com a implantação da política de incentivos fiscais para a atividade florestal, foram reflorestados cerca de 300.000 ha apenas no Rio Grande do Sul. Esses plantios, além de distantes dos centros consumidores, foram efetuados sem planejamento adequado da produção, resultando em povoamentos com árvores finas e com grande quantidade de nós. Dessa forma, em função da baixa qualidade, os produtos oriundos dessas florestas têm sua utilização e valor de mercado reduzidos.

Com o fim dos incentivos governamentais, que perduraram até meados de 1988, tornou-se necessária a adequação de técnicas para um aumento na produtividade e na qualidade dos povoamentos. Desse modo os produtos oriundos desses povoamentos, embora com maiores custos, teriam condições de competir no mercado em níveis atraentes para os investidores. Uma das formas de se alcançar esse objetivo e aumentar o valor da produção é a aplicação de tratos culturais intensivos e a antecipação dos cortes.

Conforme ZOBEL (1981), grande parte da madeira produzida no futuro será de plantações de rápido crescimento. Essa madeira é diferente daquela produzida em povoamentos naturais mais velhos da mesma espécie por apresentar uma alta proporção de lenho juvenil - pois as árvores atingem precocemente grandes diâmetros e são cortadas ainda jovens. A madeira de povoamentos jovens não é necessariamente inferior. Sua utilidade será função do produto final desejado.

Além da presença de lenho juvenil, outro problema encontrado ao se trabalhar com madeira reflorestada é a significativa ocorrência de defeitos, tais como os nós, que prejudicam as propriedades físicas e mecânicas do produto madeira. Uma forma de melhorar a qualidade da madeira, reduzindo o número de nós, é induzir a desrama natural através da utilização de espaçamentos apertados. As espécies de Pinus introduzidas no Brasil apresentam desrama natural pobre, tornando necessária a utilização da desrama artificial como meio de produzir rapidamente árvores de maiores diâmetros, com madeira de melhor qualidade.

A desrama torna possível evitar a formação de nós mortos, reduzir o diâmetro do núcleo enodado e diminuir as condições que favorecem o adelgaçamento do fuste. Apesar das vantagens 
citadas, esta é uma técnica tida como de alto custo. Por isso requer a avaliação dos seus efeitos na qualidade da madeira, de forma a justificar o investimento.

A crescente preocupação com a qualidade da produção justifica-se pelas reiteradas exigências de um mercado consumidor cada vez mais atento aos produtos que adquire. Isso força as indústrias e, conseqüentemente, os fornecedores de matéria-prima a um aprimoramento constante, que torne possível colocar no mercado produtos compatíveis com as necessidades e exigências dos consumidores.

Os objetivos da presente pesquisa foram:

a) Verificar a influência da desrama sobre fatores que influem na qualidade da madeira;

b) Caracterizar as peças em relação à porcentagem de lenho tardio;

c) Avaliar a quantidade e dimensão dos nós presentes nas peças.

\section{REVISÃO DE LITERATURA}

A densidade é um dos principais fatores usados na classificação da madeira em classes de qualidade por sua alta correlação com a resistência mecânica. Segundo KOLLMANN \& CÔTÉ (1968) as variações na densidade da madeira são devidas principalmente a diferenças na estrutura, que é caracterizada pela soma proporcional dos diferentes tipos celulares, como as fibras, traqueídeos, vasos, canais resiníferos, raios e por suas dimensões, especialmente a espessura das paredes celulares.

A posição no tronco tem um considerável efeito na densidade da madeira e, geralmente, a base tem madeira de maior densidade que a parte superior. Para Pinus spp., as variações em densidade no sentido longitudinal da árvore podem ser acentuadas a ponto de determinar diferentes classes de qualidade apenas em função da posição de origem da peça de madeira. Volkert apud KOLLMANN \& CÔTÉ (1968), observou que a densidade na base de árvores com tronco cilíndrico é maior do que nas que apresentam tronco fortemente afilado e, ainda, que as variações em densidade através de uma determinada seção transversal do tronco são menos pronunciadas que as variações em altura, sendo muitas vezes afetadas pela largura dos anéis de crescimento ou pela proporção de lenho tardio.

Esse mesmo autor descreve que o contraste em densidade entre os lenhos inicial e tardio é mais pronunciado em coníferas e, de forma geral, a densidade do lenho inicial decresce da medula para a casca e tende a diminuir da base para o topo. Por outro lado, a densidade do lenho tardio aumenta da medula para a casca e decresce da base para o topo. Considera-se que em coníferas a variabilidade em densidade depende menos da variabilidade em densidade dos lenhos inicial e tardio do que da variabilidade da porcentagem de lenho tardio. Muniz apud RINCOSKI (1994), trabalhando com Pinus taeda e Pinus elliottii, observou que os primeiros anéis de crescimento formados em árvores de rápido crescimento, na direção medula-casca, são bastante largos com a faixa de lenho tardio apresentando-se bastante estreita. Porém, à medida que se aproxima da casca, a 
porcentagem de lenho tardio aumenta de maneira significativa, e esse fato é ainda mais acentuado na base da árvore. Para Pinus taeda, Bethel apud JANE (1970) encontrou que para uma melhor resistência à compressão paralela à grã a porcentagem ótima de lenho tardio deve ser de aproximadamente $48 \%$.

A largura dos anéis de crescimento é uma característica freqüentemente associada à resistência da madeira. Porém, de acordo com Knigge \& Schulz apud DURLO (1988), essa pode não ser uma escala segura para estimar a resistência da madeira, sendo válida somente pelo fato de que anéis mais estreitos apresentam normalmente maior proporção de lenho tardio e, esse sim, é o responsável direto pela maior densidade e maior resistência da madeira. BARRICHELLO \& BRITO (1979) verificaram que a densidade do lenho tardio de Pinus caribea na região de Agudos (SP) é de 30 a $100 \%$ maior que a densidade do lenho inicial e, como existe forte correlação entre a porcentagem de lenho tardio e a densidade, é de se esperar que quanto maior for a porcentagem do primeiro, maior será a resistência da madeira.

Taras apud RINCOSKI (1994), durante estudos realizados com propriedades da madeira de Pinus elliottii observou que a densidade do lenho inicial diminui com o aumento da idade, estabilizando-se entre os 8 e os 12 anos. Já a densidade do lenho tardio aumentou rapidamente nesse mesmo período de crescimento, estabilizando-se em determinada idade, para então diminuir em direção à casca.

De forma geral, pode-se afirmar que as três maiores causas de perdas em madeiras jovens são o tamanho e a freqüência dos nós, lenho de reação e grã espiralada. De acordo com DANIEL (1979), qualquer medida que seja tomada para minimizar o efeito desses fatores trará aumentos substanciais no valor da produção dos povoamentos florestais. Assim, de forma geral, a qualidade da madeira pode ser influenciada por tratamentos que afetem o espaçamento, a proporção de copa e a taxa de crescimento.

O controle do espaçamento permite exercer influência direta sobre três importantes critérios de qualidade: o tamanho dos nós, a proporção de lenho juvenil e a forma do fuste. De todos os critérios de qualidade da madeira o tamanho dos ramos é o menos herdável, já que é muito mais afetado pelo ambiente ou pelo espaço disponível para desenvolvimento.

A proporção de copa viva pode ser controlada diretamente pela desrama, uma prática estreitamente relacionada com a qualidade da madeira. A copa viva da árvore define a posição em que as auxinas e carboidratos são produzidos, e a presença e relativa abundância desses materiais tem forte influência na extensão do lenho juvenil, na proporção de lenho inicial e tardio e na posição no tronco da máxima largura de anel, definindo o afilamento do tronco (DANIEL, 1979).

Conforme HAWLEY \& SMITH (1972), o valor e a utilidade da madeira de povoamentos manejados são reduzidos mais pelos nós e pelas distorções da grã do que por qualquer outro fator. Os ramos, depois de findarem sua atividade fisiológica, raramente caem, pois sua presença não constitui uma desvantagem particular para a sobrevivência da árvore. Assim, a desrama artificial é realizada com o intuito de aumentar a qualidade do produto final, obtendo-se madeira limpa em partes do tronco que de outra forma só produziriam material de classes inferiores.

Segundo os mesmos autores, os desbastes tendem a deter a desrama natural e estimular o 
desenvolvimento de ramos mais grossos e, se não for realizada a desrama artificial, o tamanho dos nós será aumentado. Deve-se considerar que a velocidade com que morrem os ramos mais baixos é determinada em grande parte pela densidade inicial do povoamento. Em geral, quando as copas das árvores começam a se tocar, os ramos que se encontram abaixo do ponto de intersecção das copas perdem vigor e morrem. Por sua vez, o número de árvores do povoamento determina também o diâmetro alcançado pelos ramos antes de morrerem, o que irá influenciar a velocidade de desprendimento e a qualidade da madeira no núcleo nodoso formado antes de se completar a poda natural. Assim, se o objetivo é a produção de uma grande quantidade de madeira limpa sem recorrer à desrama artificial deve-se atrasar os desbastes até que a desrama natural tenha atingido a altura desejada.

\section{MATERIAL E MÉTODOS}

\section{Características do povoamento e área}

A floresta em estudo é formada por plantio homogêneo de Pinus elliottii Engelman, do projeto Todeschini IV de propriedade da empresa Todeschini S.A., situada na localidade de Cordilheira, Serra do Piquiri, no extremo sul do município de Cachoeira do Sul (RS). A área está delimitada pelas coordenadas geográficas 30 e $31^{0}$ de latitude sul e 52 e $53^{0}$ de longitude oeste. O povoamento onde foram coletados os dados foi implantado entre julho e agosto de 1984, com espaçamento inicial de $3 \times 2 \mathrm{~m}$.

\section{Características edafoclimáticas}

Conforme MORENO (1961), o clima da região é do tipo $\mathrm{Cfb} / \mathrm{g}$, definido pela temperatura média do mês mais quente de $24,8^{0} \mathrm{C}$ e a do mês mais frio de $13,6^{0} \mathrm{C}$. As chuvas estão distribuídas por todos os meses do ano, sendo junho o mês mais chuvoso, com $166 \mathrm{~mm}$, e dezembro o mês de menor ocorrência de chuvas, com $94 \mathrm{~mm}$. A região apresenta um deficit hídrico médio de $85 \mathrm{~mm}$, distribuídos de dezembro a março. A umidade relativa é em média de $82 \%$ no inverno e $87 \%$ no verão, ficando a média anual em torno de $81 \%$.

A formação de geadas é freqüente, com uma média de 22 ocorrências por ano, e os ventos mais comuns são do sudeste e do leste, esse último predominante no mês de abril. A altitude média da região varia entre 100 e 500 m e o relevo é ondulado, com formação de vales abertos.

O solo da área experimental faz parte da unidade de mapeamento Pinheiro Machado que se caracteriza por apresentar solos litólicos, bem drenados, de coloração escura, textura média, com percentagens elevadas das frações mais grosseiras (areia grossa e cascalhos), tendo como material de origem granitos. (LEMOS et al., 1973).

\section{Características do experimento}

O experimento foi instalado na borda de um vale aberto, com parcelas de $400 \mathrm{~m}^{2}(20 \times 20$ 
m), utilizando-se o delineamento em blocos casualizados, com 3 repetições. As parcelas têm bordaduras intra-blocos de 3 linhas de árvores e os blocos bordaduras de 4 árvores $(8 \mathrm{~m})$.

Os tratamentos utilizados foram: $\mathrm{T} 1=$ sem desrama (testemunha); $\mathrm{T} 2=$ desrama seca; $\mathrm{T} 3=$ desrama de $40 \%$ da altura total; T4 $=$ desrama de $50 \%$ da altura total; T5 = desrama de $60 \%$ da altura total.

Desde a implantação do experimento, em 1989, foram realizadas 5 operações de desrama, nos anos de 1989, 91, 92, 93 e 94. Para serem desramadas as árvores apresentaram diâmetro de colo superior a $5 \mathrm{~cm}$ e os tratamentos foram aplicados até as árvores atingirem 6 metros de fuste desramado.

A partir dos tratamentos aplicados nas unidades amostrais, foram estudadas as influências destes sobre as seguintes variáveis respostas:

a) proporção de lenho tardio;

b) quantidade e dimensão de nós.

\section{Obtenção dos dados}

Os ensaios foram realizados em material proveniente do primeiro desbaste do povoamento, realizado aos 10 anos. De cada uma das unidades amostrais foram retiradas três entre as maiores árvores marcadas para desbaste, totalizando 45 árvores-amostra nas 3 repetições.

De cada árvore foram retiradas as duas primeiras toras, com 2,5 metros de comprimento cada uma. Inicialmente foi retirado um pranchão central de cada uma das toras, com 2 polegadas de espessura, que foi seco em estufa convencional. Esses pranchões foram transformados em corpos de prova, com dimensões nominais de 2" $x$ 4" $x$ 8' $(0,05 \times 0,10 \times 2,5 \mathrm{~m})$ nos quais foram determinada as quantidade e tamanho dos nós. Também foram retirados discos (secções transversais) - dois em cada árvore -, um na base e outro a 2,5 m aproximadamente, para análise da proporção de lenho tardio.

\section{Análise estatística}

$\mathrm{Na}$ análise da variância foram utilizados dois contrastes para a comparação das médias de tratamentos (T1 vs. T2,T3,T4 e T5 e, ainda, T2 vs. T3, T4 e T5) e análise de regressão. O quadro de análise da variância, com a decomposição da causa de variação "tratamentos" foi definido conforme mostra a Tabela 1.

As hipóteses testadas para os contrastes ortogonais foram:

$\mathrm{H}_{0}$ : o contraste é nulo $\left(\mathrm{X}_{\mathrm{r}}=0\right)$

$\mathrm{H}_{1}: \mathrm{X}_{\mathrm{r}} \neq 0$

O critério de decisão utilizado foi:

Se $F_{(X r)}>F_{\alpha(1 ; G L E)}$, rejeita-se $H_{0}$, 
Se $\mathrm{F}_{(\mathrm{Xr})}<\mathrm{F}_{\alpha(1 ; \mathrm{GLE})}$, não rejeita-se $\mathrm{H}_{0}$.

TABELA 1: Discriminação da análise da variância do experimento.

\begin{tabular}{lc}
\hline Causas de Variação (CV) & Graus de Liberdade (GL) \\
\hline Blocos & 2 \\
Tratamentos & 4 \\
X1 (T1 x T2,T3,T4,T5) & 1 \\
X2 (T2 x T3,T4,T5) & 1 \\
Regressão linear & 1 \\
Regressão quadrática & 1 \\
Erro experimental & 10 \\
Erro amostral & 30 \\
\hline Total & 46 \\
\hline
\end{tabular}

\section{Determinação da quantidade de nós e da percentagem de lenho tardio}

A determinação da quantidade de nós foi feita em percentagem do volume total das peças. Para isso determinou-se o volume de cada um dos nós encontrados nas peças.

A percentagem de lenho tardio foi determinada para cada tratamento através da medição dos anéis de crescimento dos discos. Inicialmente estabelem-se diversos raios e, ao longo desses raios, fez-se a medição do lenho tardio nos anéis de crescimento. Assim, a proporção de lenho tardio foi obtida através da expressão:

$\mathrm{LO}(\%)=\left(\sum\right.$ dos comprimentos de Lenho Tardio $) /($ Comprimento do Ráio $) .100$

Como a amostragem foi relativamente pequena, por conveniência, conforme recomendado por FREESE (1970), a variável dependente foi transformada para $y=\operatorname{arco}$ seno $\sqrt{\circ}$ de LO.

\section{RESULTADOS E DISCUSSÃO}

\section{Proporção de lenho tardio}

O ajuste de uma regressão quadrática aos dados de percentagem de lenho tardio da primeira e segunda toras de Pinus elliottii nos diferentes tratamentos (Tabelas 2 e 3) não mostrou significância ao nível de $5 \%$ de probabilidade.

Os valores calculados de F, encontrados para a primeira e segunda toras foram, respectivamente, de 1,16 e 0,03 , e o valor tabelado de $\mathrm{F}_{5 \%(2,9)}$ é de 4,26. Também não foi encontrada regressão linear significativa, ao nível de significância de 5\%. Os valores de $\mathrm{F}$ calculados foram de 1,47 para a primeira tora e de 0,03 para a segunda, enquanto o valor tabelado de $F_{5 \%(1,10)}$ foi de 4,96 . 
TABELA 2: Percentagem de lenho tardio na $1^{\underline{a}}$ tora.

\begin{tabular}{l|ccc}
\hline \multirow{2}{*}{ Tratamentos } & \multicolumn{3}{c}{ \% de lenho tardio } \\
\cline { 2 - 4 } & Bloco 1 & Bloco 2 & Bloco 3 \\
\hline T1 - Sem desrama (s/d) & 29,36 & 29,30 & 27,69 \\
T2 - Desrama seca (ds) & 29,42 & 29,85 & 32,59 \\
T3 - 40\% & 31,37 & 29,69 & 29,14 \\
T4 - 50\% & 30,39 & 32,32 & 31,28 \\
T5 - 60\% & 26,51 & 30,55 & 31,64 \\
\hline
\end{tabular}

TABELA 3: Percentagem de lenho tardio na $2^{\underline{a}}$ tora.

\begin{tabular}{l|c|c|c}
\hline \multirow{2}{*}{ Tratamentos } & \multicolumn{3}{|c}{ \% de lenho tardio } \\
\cline { 2 - 4 } & Bloco 1 & Bloco 2 & Bloco 3 \\
\hline T1 - Sem desrama (s/d) & 24,95 & 26,65 & 26,45 \\
T2 - Desrama seca (ds) & 27,01 & 26,30 & 26,24 \\
T3 - 40\% & 26,18 & 25,30 & 24,26 \\
T4 - 50\% & 27,43 & 26,71 & 26,98 \\
T5 - 60\% & 23,14 & 27,25 & 25,32 \\
\hline
\end{tabular}

Da mesma forma, os contrastes ortogonais $\left(\mathrm{X}_{1}=\mathrm{T} 1 x \mathrm{~T} 2, \mathrm{~T} 3\right.$, T4 e T5; e $\mathrm{X}_{2}=\mathrm{T} 2 x \mathrm{~T} 3, \mathrm{~T} 4$ e T5) descritos nas Tabelas 4 e 5, respectivamente para as peças da primeira e segunda toras das árvores-amostra, não apresentaram resultado significativo como pode ser observado na Tabela 6.

TABELA 4: Contrastes ortogonais aplicados às médias de $\%$ de lenho tardio da $1^{1}$ tora.

\begin{tabular}{l|c|c|c|c|c}
\hline $\mathbf{t}_{\mathbf{i}}$ & $\mathbf{Y}_{\mathbf{i} .}$ & $\mathbf{C}_{\mathbf{1}}$ & $\mathbf{C}_{\mathbf{2}}$ & $\mathbf{C}_{\mathbf{1}} \mathbf{Y}_{\mathbf{i} .}$ & $\mathbf{C}_{\mathbf{2}} \mathbf{Y}_{\mathbf{i} .}$ \\
\hline $\mathrm{T} 1$ & 28,78 & -4 & 0 & $-115,12$ & 0,00 \\
$\mathrm{~T} 2$ & 30,62 & 1 & -3 & 30,62 & $-91,86$ \\
$\mathrm{~T} 3$ & 30,06 & 1 & 1 & 30,06 & 30,06 \\
$\mathrm{~T} 4$ & 31,33 & 1 & 1 & 31,33 & 31,33 \\
$\mathrm{~T} 5$ & 29,56 & 1 & 1 & 29,56 & 29,56 \\
\hline$\Sigma$ & - & - & - & 6,45 & $-0,91$ \\
\hline
\end{tabular}

$\mathrm{O}$ valor de $\mathrm{F}_{5 \%(1, \mathrm{GLE})}$, tabelado para $5 \%$ de probabilidade, para 1 grau de liberdade do contraste e 8 graus de liberdade do erro é de 5,32, superior aos valores apresentados para a $1^{\mathrm{a}}$ e $2^{\mathrm{a}}$ toras. Dessa forma, rejeita-se a hipótese $\mathrm{H}_{0}$, não existindo diferenças significativas entre as médias dos tratamentos.

Na Figura 1 encontram-se representados os valores médios de percentagem de lenho tardio nos diferentes tratamentos. Na primeira tora, o tratamento 4 apresentou a maior média de percentagem de lenho tardio, de 31,33\%. Já o tratamento 1 apresentou, em média, 28,78\% de lenho tardio, valor um pouco inferior ao encontrado para os demais tratamentos, entre 29,5 e $30,6 \%$. As peças provenientes da segunda tora apresentaram, conforme esperado, valores de percentagem de lenho tardio inferiores aos encontrados nas peças da primeira tora. $\mathrm{O}$ tratamento 4, onde as árvores são desramadas até $50 \%$ de sua altura, foi o que apresentou a maior média, de $27,04 \%$. Porém os 
demais tratamentos apresentam valores apenas um pouco inferiores a esse, entre $25 \%$ nos tratamentos 3 e 5 e $26 \%$ nos tratamentos 1 e 2 .

TABELA 5: Contrastes ortogonais aplicados às médias de $\%$ de lenho tardio da $2^{-a}$ tora.

\begin{tabular}{cccccccc}
\hline $\mathbf{t}_{\mathbf{i}}$ & $\mathbf{Y}_{\mathbf{i} .}$ & $\mathbf{C}_{\mathbf{1}}$ & $\mathbf{C}_{\mathbf{2}}$ & $\mathbf{C}_{\mathbf{1}} \mathbf{Y}_{\mathbf{i} .}$ & $\mathbf{C}_{\mathbf{2}} \mathbf{Y}_{\mathbf{i} .}$ \\
\hline T1 & 26,02 & -4 & 0 & $-104,08$ & 0,00 \\
$\mathrm{~T} 2$ & 26,52 & 1 & -3 & 26,52 & $-79,56$ \\
$\mathrm{~T} 3$ & 25,25 & 1 & 1 & 25,25 & 25,25 \\
$\mathrm{~T} 4$ & 27,04 & 1 & 1 & 27,04 & 27,04 \\
$\mathrm{~T} 5$ & 25,24 & 1 & 1 & 25,24 & 25,24 \\
\hline$\Sigma$ & - & - & - & $-0,03$ & $-2,03$ \\
\hline
\end{tabular}

TABELA 6: Valores de $\mathrm{F}_{(\mathrm{X})}$ para os contrastes aplicados às médias de $\%$ de lenho tardio.

\begin{tabular}{l|c|c}
\hline Contrastes & $\mathbf{1}^{\mathbf{a}}$ tora & $\mathbf{2}^{\mathbf{a}}$ tora \\
\hline $\mathrm{F}_{(\mathrm{X} 1)}$ & 2,12 & $7,6 \times 10^{-5}$ \\
$\mathrm{~F}_{(\mathrm{X} 2)}$ & 0,07 & 0,73 \\
\hline
\end{tabular}

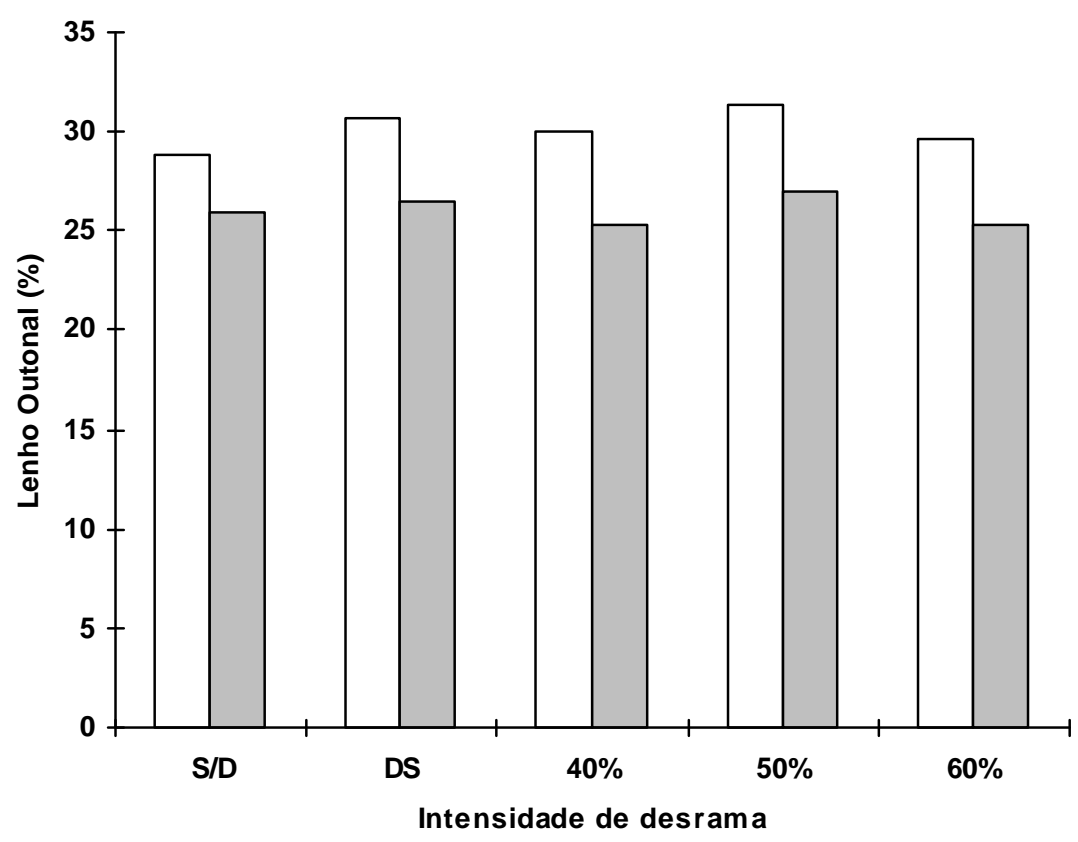

$\square$ 1a. peça $\square$ 2a. peça

FIGURA 1: Valores médios da percentagem de lenho tardio.

\section{Quantidade de nós}

Os valores referentes à quantidade de nós encontrados nas peças avaliadas, expressos em percentagem de volume total, encontram-se nas Tabelas 7, para a primeira e segunda toras. 
TABELA 7: Quantidade de nós (\%) na primeira e segunda tora das árvores-amostra

\begin{tabular}{l|ccc|c|c|c}
\hline \multirow{2}{*}{ Tratamentos } & \multicolumn{6}{|c}{ \% de nós } \\
\cline { 2 - 7 } & \multicolumn{3}{|c|}{$1^{\text {a }}$ tora } & \multicolumn{3}{c}{$2^{\text {a }}$ tora } \\
\cline { 2 - 7 } & Bloco 1 & Bloco 2 & Bloco 3 & Bloco 1 & Bloco 2 & Bloco 3 \\
\hline T1 - Sem desrama (s/d) & 0,48 & 0,34 & 0,39 & 0,68 & 0,42 & 0,27 \\
T2 - Desrama seca (ds) & 0,38 & 0,33 & 0,31 & 0,39 & 0,65 & 0,45 \\
T3 - 40\% & 0,41 & 0,36 & 0,29 & 0,47 & 0,42 & 0,23 \\
T4 - 50\% & 0,31 & 0,39 & 0,32 & 0,38 & 0,48 & 0,40 \\
T5 - 60\% & 0,52 & 0,27 & 0,29 & 0,56 & 0,54 & 0,34 \\
\hline
\end{tabular}

A regressão quadrática testada para descrever a percentagem de nós nas toras não foi significativa, apresentando um valor calculado de $\mathrm{F}$, para a primeira e segunda toras de, respectivamente, 0,48 e 0,57, enquanto o valor tabelado de $\mathrm{F}_{5 \%(2,9)}$ é de 4,26, para 5\% de probabilidade. Da mesma forma, não foi encontrada regressão linear significativa entre os dados de percentagem de nós. $\mathrm{O}$ valor calculado de $\mathrm{F}$ foi de 0,84 para a primeira tora e de 0,002 para a segunda, enquanto o valor tabelado de $\mathrm{F}_{5 \%(1,10)}$ foi de 4,96 , para $5 \%$ de probabilidade.

Os contrastes ortogonais aplicados para comparação das médias de quantidade de nós dos tratamentos encontram-se descritos, para a primeira e segunda toras, nas Tabelas 8 e 9, respectivamente.

TABELA 8: Contrastes ortogonais aplicados às médias de percentagem de nós das peças da $1^{\text {a }}$ tora.

\begin{tabular}{lcccccc}
\hline $\mathbf{t}_{\mathbf{i}}$ & $\mathbf{Y}_{\mathbf{i} .}$ & $\mathbf{C}_{\mathbf{1}}$ & $\mathbf{C}_{\mathbf{2}}$ & $\mathbf{C}_{\mathbf{1}} \mathbf{Y}_{\mathbf{i} .}$ & $\mathbf{C}_{\mathbf{2}} \mathbf{Y}_{\mathbf{i} .}$ \\
\hline $\mathrm{T} 1$ & 0,403 & -4 & 0 & $-1,61$ & 0,00 \\
$\mathrm{~T} 2$ & 0,340 & 1 & -3 & 0,34 & $-1,02$ \\
$\mathrm{~T} 3$ & 0,353 & 1 & 1 & 0,35 & 0,35 \\
$\mathrm{~T} 4$ & 0,340 & 1 & 1 & 0,34 & 0,34 \\
$\mathrm{~T} 5$ & 0,360 & 1 & 1 & 0,36 & 0,36 \\
\hline$\Sigma$ & - & - & - & $-0,22$ & 0,03 \\
\hline
\end{tabular}

TABELA 9: Contrastes ortogonais aplicados às médias de percentagem de nós das peças da $2^{\mathrm{a}}$ tora.

\begin{tabular}{l|cccccc}
\hline $\mathbf{t}_{\mathbf{i}}$ & $\mathbf{Y}_{\mathbf{i} .}$ & $\mathbf{C}_{\mathbf{1}}$ & $\mathbf{C}_{\mathbf{2}}$ & $\mathbf{C}_{\mathbf{1}} \mathbf{Y}_{\mathbf{i} .}$ & $\mathbf{C}_{\mathbf{2}} \mathbf{Y}_{\mathbf{i} .}$ \\
\hline T1 & 0,46 & -4 & 0 & $-1,84$ & 0,00 \\
T2 & 0,50 & 1 & -3 & 0,50 & $-1,50$ \\
T3 & 0,38 & 1 & 1 & 0,38 & 0,38 \\
T4 & 0,42 & 1 & 1 & 0,42 & 0,42 \\
T5 & 0,48 & 1 & 1 & 0,48 & 0,48 \\
\hline$\Sigma$ & - & - & - & $-0,06$ & $-0,22$ \\
\hline
\end{tabular}

$\mathrm{O}$ valor de $\mathrm{F}_{5 \%(1, \mathrm{GLE})}$, tabelado para $5 \%$ de probabilidade, para 1 grau de liberdade do contraste e 8 graus de liberdade do erro é de 5,32. Como pode ser observado na Tabela 10, os contrastes aplicados não foram significativos, rejeitando-se a hipótese $\mathrm{H}_{0}$, pois não existem 
diferenças significativas entre as médias de quantidade de nós dos tratamentos testados.

TABELA 10: Valores de $\mathrm{F}_{(\mathrm{X})}$ para os contrastes aplicados às médias de percentagem de nós.

\begin{tabular}{l|c|c}
\hline Contrastes & $\mathbf{1}^{\text {a }}$ tora & $\mathbf{2}^{\text {a }}$ tora \\
\hline $\mathrm{F}_{(\mathrm{X} 1)}$ & 1,71 & 0,04 \\
$\mathrm{~F}_{(\mathrm{X} 2)}$ & 0,07 & 0,90 \\
\hline
\end{tabular}

Os valores médios de quantidade de nós para as peças avaliadas nos tratamentos estudados são apresentados na Figura 2. De acordo com a figura, nas peças da primeira tora, o tratamento 1 apresentou a maior média de percentagem de nós: $0,40 \%$, enquanto a menor média foi de $0,34 \%$, nos tratamentos 2 e 4 . Os tratamentos 3 e 5 tiveram, respectivamente, percentagens médias de nós 0,35 e $0,36 \%$. Para as peças da segunda tora foi encontrada uma maior variação entre as médias dos tratamentos. A maior média ocorreu no tratamento 2 , de $0,50 \%$, e a menor foi encontrada no tratamento 3 , de $0,37 \%$. Os tratamentos 1,4 e 5 tiveram, respectivamente, médias de $0,46,0,42$ e $0,48 \%$.

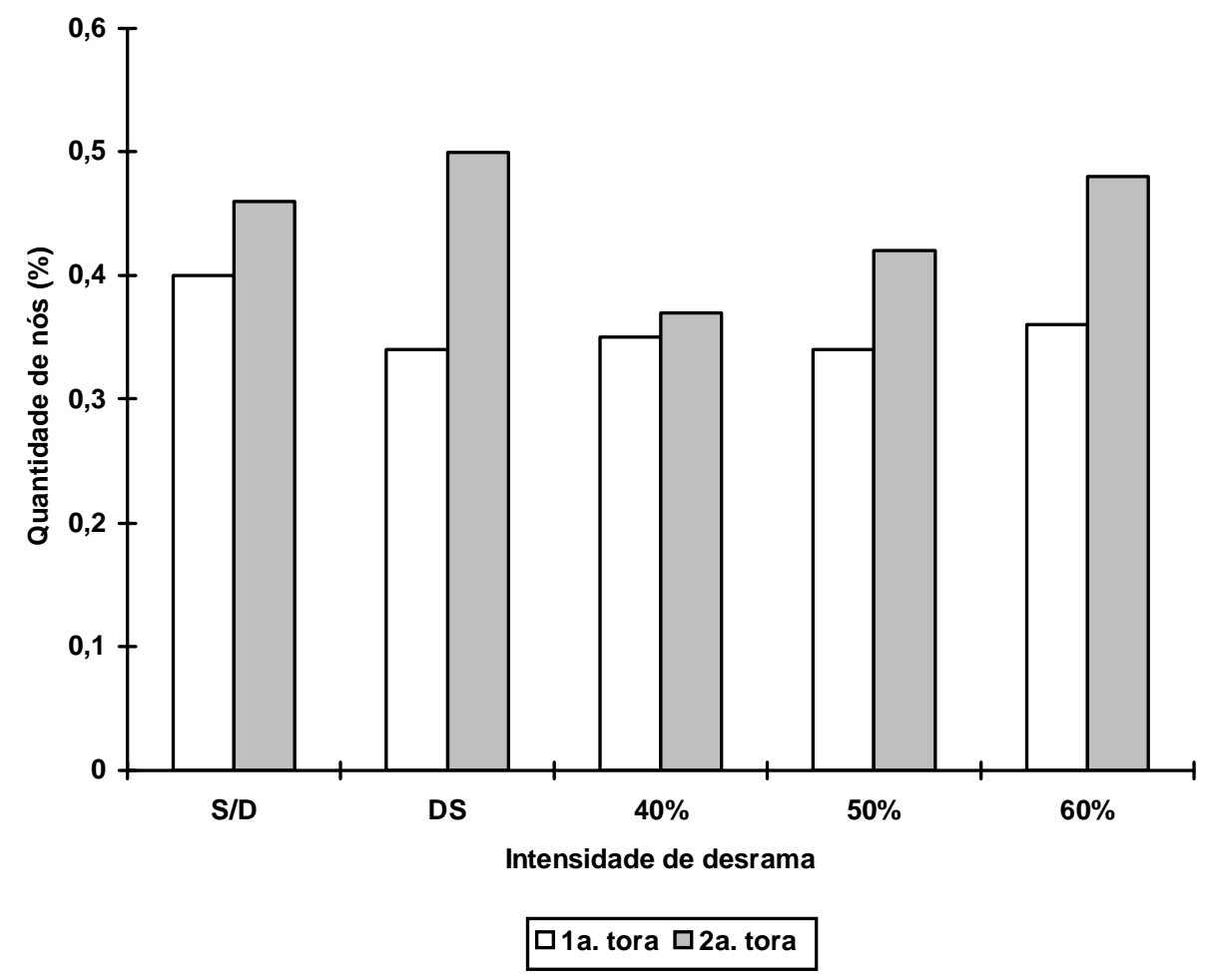

FIGURA 2: Valores médios de percentagem de nós na primeira e segunda toras 


\section{CONCLUSÃO}

Considerando-se os resultados obtidos, pode-se concluir que:

a) Para a proporção de lenho tardio, na primeira e segunda toras, não foi encontrada regressão significativa. Da mesma forma, não foi constatada diferença significativa entre as médias dos tratamentos. Na primeira tora, o tratamento T4 apresenta a maior média, de 31,33\%; já o tratamento T1 apresenta o menor valor médio, $28,78 \%$. Nas peças da segunda tora, a maior média ocorreu no tratamento $\mathrm{T} 4$, de $27,04 \%$, enquanto os demais tratamentos apresentam valores médios de lenho tardio entre 25 e $26 \%$.

b) Para a quantidade de nós, expressa na forma de porcentagem de volume total das peças, não se obteve regressão significativa. A comparação das médias dos tratamentos também não evidenciou diferenças significativas. Nas peças da primeira tora, o tratamento $\mathrm{T} 1$ apresentou a maior quantidade média de nós, cerca de $0,40 \%$, enquanto os tratamentos T2 e T4 apresentaram em média 0,34\% de nós. Para a segunda tora, o tratamento T2 foi o que apresentou maior porcentagem de nós em suas peças, cerca de $0,50 \%$, enquanto o tratamento T3 apresentou o menor valor, de $0,37 \%$.

\section{BIBLIOGRAFIA CONSULTADA}

BARRICHELO, L. E. G.; BRITO, J. O. Variabilidade radial da madeira de Pinus caribea var. hondurensis. IPEF, n. 18, p. 81-102, 1979.

DANIEL, T. W. Principles of silviculture. 2.ed. New York:McGraw-Hill, 1979.

DURLO, M. A. Determinação das variáveis para a caracterização de Pinus elliottii com finalidade estrutural. In: CONGRESSO FLORESTAL ESTADUAL, Nova Prata, 1988. Anais. Vol. 2, p. 997-1111.

FREESE,F. Metodos estadisticos elementales para tecnicos forestales. Mexico:Agencia para el desarrollo internacional, 1970. 104 p.

JANE, F. W. The structure of wood. London:Adam \& Charles Black, 1970. 2.Ed.

HAWLEY, R. C.; SMITH, D. M. Silvicultura práctica. Barcelona: Omega, 1972.

KOLLMANN, F. F. P.;CÔTÉ JR, W. A. Principles of wood science and technology. I. Solid Wood. New York:Springer, 1968.

LEMOS, R. C.; AZOLIN, M. A. D.; ABRAÃO, P. V. R.; SANTOS, M. C. L. Levantamento e reconhecimento dos solos do Estado do Rio Grande do Sul. Recife: Ministério da Agricultura, Departamento Nacional de Pesquisas Agropecuárias - Divisão de Pesquisas Pedológicas, 1973. 431p. (Boletim Técnico, 30).

MOREnO, J. A. Clima do Rio Grande do Sul. Porto Alegre: Secretaria da Agricultura, 1961. 42 p. 
RINCOSKI, C. R. Efeito da resinagem nas características da madeira de Pinus elliottii Engelm var. Elliottii. Curitiba, 1994. Dissertação (Mestrado em Ciências Florestais) - Curso de PósGraduação em Engenharia Florestal, Universidade Federal do Paraná.

ZOBEL, B . Wood quality from fast-grown plantations. TAPPI, v. 64, n. 1, p. 71-74,1981. 